\title{
More on contextual effects on learning and memory
}

\author{
RICHARD A. MONTY \\ Behavioral Research Directorate, U.S. Army Human Engineering Laboratory \\ Aberdeen Proving Ground, Maryland 21005 \\ LAWRENCE C. PERLMUTER and DAVID LIBON \\ Veterans Administration Outpatient Clinic, Boston, Massachusetts 02108 \\ and \\ TERESSA BENNET \\ Virginia Polytechnic Institute and State University, Blacksburg, Virginia 24061
}

\begin{abstract}
Subjects were presented a page of words containing either two or four words per line. A single word (target) on each line was to be learned. Results showed that, as in an earlier experiment (Perlmuter \& Monty, 1982), directing attention to background words by allowing subjects to choose the target words enhanced the learning of background and target items. Moreover, when subjects were permitted to select their targets by rejecting those that they did not wish to learn, overall performance was even better than when choice was permitted. Thus, the earlier observations of the interrelatedness of target and background learning were extended and show that such joint learning can be greatly enhanced by giving the subject the opportunity to choose or reject target words. Possible reasons for these observations were discussed.
\end{abstract}

In an earlier experiment, Perlmuter and Monty (1982) found that when subjects were allowed to choose one target word from a line of words, memory for not only the chosen item but also for the nonchosen or background words was enhanced relative to a "force" condition in which no choice was offered. There are at least two explanations for that observation. First, it is possible that attention directed to the nonchosen words during the act of choosing enhanced incidental learning of the nonchosen words, which in turn enhanced memory for the target words. This is similar to Schulman's (1973) observation that learning of background information (e.g., position on a page) is associated with enhanced target recall. Alternatively, allowing the subjects to choose materials they wish to learn might have resulted in a generalized state of motivation, which in turn resulted in better learning of both the target (chosen) and background (nonchosen or force) words. This mechanism has been discussed more fully by Monty,

This research was supported in part by grants from the U.S. Army Human Engineering Laboratory Behavioral Research Directorate and the Veterans Administration. Thanks go to Janet England and Alan Feiner for assistance with data analyses. Victor Hoffman provided assistance with computer programs and analyses; his help is gratefully acknowledged. Robert A. Goodale of Boston State College graciously provided laboratory space and access to subjects. Special thanks go to Deborah Birkmire, Malekah Hakami, Robert Karsh, Wayne Shebilske, and Harvey A. Taub for constructive reviews of earlier versions of this manuscript. Address requests for reprints to Richard A. Monty, Behavioral Research Directorate, U.S. Army Human Engineering Laboratory, Aberdeen Proving Ground, Maryland 21005 .
Geller, Savage, and Perlmuter (1979) and Perlmuter and Monty (1977).

In the present experiment, two additional conditions were introduced to the Perlmuter and Monty (1982) paradigm to more precisely evaluate differential attention to the background items. One of these conditions required the subjects to reject the nondesired (background) alternatives they did not want to learn. In the process of rejection, attention to the background items is likely to be greater than it is when selections are made through choice. It was thus hypothesized that the act of rejection should enhance processing levels for both target and background words, thereby facilitating learning of these items relative to the choice condition. Second, a choice-modified condition was added, in which following target selection, subjects crossed out nonchosen words to insure direct contact or greater commerce with the nonchosen background words than subjects in the choice group experienced. Should these attentional manipulations affect performance as hypothesized, they would seem to favor the incidental learning hypothesis over the motivational one, which attributes enhanced performance to the perception of control that results from the act of choosing.

Finally, several procedural changes were made in the paradigm employed by Perlmuter and Monty (1982). First, whereas the earlier experiment treated choice/ force as a within-subjects variable, there was the possibility that the positive effects attributed to choice generalized to the force condition, especially if motivation was involved. In the present experiment, choice/ force was treated as a between-subjects variable, and it was hypothesized that the effects of offering a subject 
a choice would be even more apparent. Second, Perlmuter and Monty employed a target discrimination task to measure target learning, which was followed by an unanticipated recognition test to examine learning of the nonchosen background materials. In such a design, there is the possibility that the results of the second task may be contaminated by exposing the subject to the first task. Thus, in the present experiment, the unanticipated recognition task immediately followed the choice/ force procedure. Third, it was suggested in the earlier experiment that the enhanced learning of subjectselected targets could be accounted for on the basis that the subjects merely selected the items that were easiest to learn. In the present experiment, the individual force items were thus yoked to those selected by the choice subjects to eliminate the possibility that "item selection" accounts for improved performance in the choice condition. Parenthetically, it should be noted that in a number of paired associate studies (e.g., Perlmuter, Monty, \& Kimble, 1971), such a yoking procedure was employed and subject-selected pairs were nevertheless learned better than were force pairs.

\section{METHOD}

\section{Subjects}

The subjects were 80 male and female students, aged 19 22 years, from an introductory psychology class at Virginia Polytechnic Institute and State University.

\section{Procedure}

As in the Perlmuter and Monty (1982) experiment, subjects were presented five-letter words of high meaningfulness drawn from a list generated by Locascio and Ley (1972). In this experiment, however, there were only 20 lines of words, 10 with 2 items/line and 10 with 4 items/line.

Each of the four groups contained 20 subjects who were assigned at random. Subjects in the choice group initially received a practice session similar to that in the earlier experiment. Next, all of the materials were presented on a page containing the 20 lines of items, and subjects read these silently in $25 \mathrm{sec}$. Following this, the same rows of words were presented in a different order, and subjects chose and underlined the words they wished to learn within a total $55-\mathrm{sec}$ period. On each line, a single word was selected.

Following a 1-min rest period, an unanticipated and untimed recognition and confidence rating task was presented in the same manner as in the earlier experiment, except that it preceded the target discrimination task. Subjects received the 60 old words interspersed with an equal number of similar new words. This was followed by two successive 45 -sec target discrimination test trials in which, unlike in the earlier experiment, none of the alternatives was crossed out on the four-item test lines.

Subjects in the force group were treated identically, except that at the point at which the choice subjects selected their words, the force subjects were assigned words selected by a previous yoked choice subject. The target words were underlined prior to being presented, and the subjects were required to reunderline each one.

Subjects in the reject group underlined the words they did not wish to learn and were required to remember the single word on each line that remained without the underline.

In the choice-modified group, subjects selected their words as they did in the choice group and, in addition, following selection of the chosen words, they crossed out the nonchosen words. Thus, the last two groups had direct contact and presumably even greater commerce with the nonchosen (rejected) background words than did the subjects in the choice group.

\section{RESULTS}

\section{Recognition Test}

With respect to performance on the recognition task, the 60 old words (i.e., the words that had been presented during the choice/force phase) were treated as follows: A 6-point rating scale was established whereby if the subject reported "old" and "very confident," "moderately confident," or "unconfident," a rating of 6,5 , or 4 was used, respectively. If they reported "new" and "unconfident," "moderately confident," or "very confident," a rating of 3,2 , or 1 was used, respectively. Thus, the best score (6), was assigned if the subjects said "old" and "very confident," as they were correct, and the worst score (1), was assigned if they reported "new" and "very confident," as they were, of course, incorrect.

The rating scores were subjected to an analysis of variance, with group (choice, force, reject, and choice modified) as a between-subjects variable and numerosity ( 2 vs. 4 items/line) and word type (target vs. background word) as within-subjects effects.

The main effect for group was significant $[F(3,76)=$ $14.59, \mathrm{p}<.001, \mathrm{MSe}=.72]$ and revealed that the recognition scores of the choice, choice-modified, and reject groups were all similar and superior to the force group (mean ratings, respectively, of $4.75,4.80,5.04$, and 4.19). Similarly, the main effect for numerosity $[F(1,76)=7.90, p<.01, \mathrm{MSe}=.15]$ reached significance, indicating that recognition was better for words that had appeared on the two-item lines than for words on the four-item lines (mean ratings, respectively, of 4.75 and 4.63), whereas the main effect for word type $[F(1,76)=446.67, \mathrm{p}<.001, \mathrm{MSe}=.26]$ indicated that the target words were recognized better than background words (mean ratings, respectively, of 5.29 and 4.09).

Several of the interactions also reached significance. The Groups by Numerosity interaction $[\mathrm{F}(3,76)=3.18$, $\mathrm{p}<.05, \mathrm{MSe}=.15$ ] indicated that as numerosity increased, recognition performance declined for both the choice and choice-modified groups, but no such decline was observed in either the reject or force groups. Further, the performance of the force group was significantly inferior to each of the other groups. Similarly, the Numerosity by Word Type interaction $[\mathrm{F}(1,76)=10.14$, $\mathrm{p}<.01, \mathrm{MSe}=1.29]$ revealed a greater decrement in recognition scores for background than for target items as numerosity increased, a result identical to that of the Perlmuter and Monty (1982) experiment.

Of greater interest is the significant Groups by Numerosity by Word Type interaction $[\mathrm{F}(3,76)=3.94$, $\mathrm{p}<.05, \mathrm{MSe}=.13$ ], shown in Figure 1. In delineating this interaction, with respect to recognition of back- 
ground words (left-hand panel), it was found that the force group was inferior to all other groups and the reject group was superior to the choice group. Second, as numerosity increased, recognition decreased for all but the force group. Examination of the target words (right-hand panel) also showed that the force group was inferior to the other groups and, again, the reject group was superior to the choice group. Further, target recognition was independent of background numerosity, with one exception: In the reject group, target recognition increased as background numerosity increased.

In contrast to the observations of Perlmuter and Monty (1982), the choice group was superior to the force group not only on the four-item lines, but on the two-item lines as well. Further, the magnitude of the difference between the choice and force group was somewhat larger in this experiment than in Perlmuter and Monty (1982), suggesting that in the earlier experiment there may in fact have been beneficial transfer effects from the choice to the force lines.

\section{Target Discrimination}

The number of target words correctly underlined on the target discrimination task were subjected to an analysis of variance, with groups as a between-subjects effect and trials and numerosity as within-subjects effects. In contrast to the earlier experiment (Perlmuter \& Monty, 1982), none of the words was crossed out on the task, and thus there were differential guessing opportunities on the two- and four-item lines, rendering the main effect for numerosity uninterpretable. The main effect for group was significant $[F(3,76)=18.30$, $\mathrm{p}<.001, \mathrm{MSe}=7.34]$ and revealed that the choice, reject, and choice-modified groups performed at about the same level (mean number correct, respectively, of $7.89,8.16$, and 7.03) and that the force group was inferior to all of the other groups (mean number correct $=5.29)$. The main effect for trials $[F(1,76)=28.97$, $\mathrm{p}<.001, \mathrm{MSe}=1.58]$ simply indicated that recognition

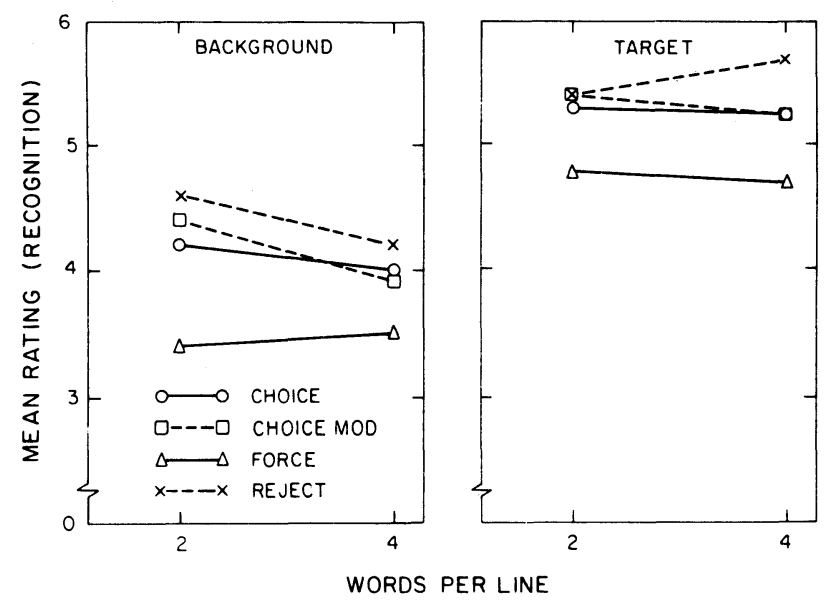

Figure 1. Mean rating on the recognition trial for target and background words as a function of the number of words per line for the choice, choice-modified, reject, and force groups.
Table 1

Mean Number of Words Correct as a Function of Groups and Number of Words per Line (WPL)

\begin{tabular}{ccccc}
\hline & \multicolumn{4}{c}{ Group } \\
\cline { 2 - 5 } WPL & Force & Choice & Reject & $\begin{array}{c}\text { Choice } \\
\text { Modified }\end{array}$ \\
\hline 2 & 6.68 & 8.68 & 8.42 & 7.80 \\
4 & 3.90 & 7.10 & 7.90 & 6.25 \\
\hline
\end{tabular}

for target items improved across trials. Finally, the Group by Numerosity interaction $[\mathrm{F}(3,76)=5.30$, $\mathrm{p}<.01, \mathrm{MSe}=3.19]$ reflected a significant decrement in target recognition with an increase in numerosity for all groups but the reject group, as shown in Table 1 . Further, as the number of words per line increased, the decrement in performance of the force group was nearly twice that of the other groups. Basically, the results of this analysis reflect those seen with the recognition data. Target recognition was best for the reject group, and the choice and choice-modified groups outperformed the force group.

\section{DISCUSSION}

We have seen that when choice or some variant of choice was permitted, performance was enhanced relative to the force group for both target and background words. Increased attention to background words may in part be responsible for improved performance on target words. Schulman (1973) found that target recall was improved if the subject incidentally acquired information about its spatial location. Analogously, in the present experiment, background words may be considered as attributes of target words. Hence, improved recognition of background words in the various conditions in which choice was permitted might be expected to contribute to improved target performance.

With respect to recognition of target items, it is clear that as attention increased to all of the items within a line, target recognition improved. For the force group, attention to background items presumably was minimal and target recognition was also poorest. In the choice group, an intermediate amount of contact with the words on the line would be anticipated. That is, in making a selection, subjects were free to consider as few or as many items on each line as they desired; hence, contact with words, on the average, should have been at an intermediate level, and the target recognition scores reflect this. The group for whom item contact was, by necessity, maximal is the one in which subjects rejected potential targets, thereby selecting the better or the best option, as would be the case on two- and fouritem lines, respectively. In this condition, the highest level of target recognition resulted. This observation not only provides additional support for the attentional hypothesis previously discussed but, more important, lays to rest the possibility that item selection was solely responsible for enhanced performance in the various choice conditions. That is, in any comparison between a choice and a force condition, it could be argued that when provided with the opportunity to learn words of their own choosing, subjects simply selected materials that were easiest for them to learn, and thus the benefits of choice are reducible to idiosyncratic choice factors that are unique to each subject. By comparison, the assignment of targets to subjects is generally incompatible with idiosyncratic factors, and thus performance is degraded. The conditions of the present experiment eliminate this potential source of bias, since in both the choice and reject 
conditions, as well as in the choice-modified condition, subjects were permitted to select targets of their own choosing. Nevertheless, the reject condition resulted in better performance than the choice condition, thus offering support for an attentional mechanism that presumably, functions more effectively following rejection than following choice. Finally, although the choicemodified group had contact with all of the items on each line, the performance of this group was inferior to that of the reject group. Apparently, simply crossing out nonchosen alternatives is not equivalent to rejecting alternatives in the process of making a selection.

\section{Conclusion}

In summary, when targets were assigned, learning was poorer than when subjects were permitted to select some of the targets for themselves. Poorer learning of experimenter-assigned targets may result, in part, from lower levels of attention to background items. On the other hand, when subjects chose their own targets, contact with and attention to background items are necessarily increased. From one perspective, this finding was somewhat surprising. That is, if it is assumed that attention to background items would diffuse or scatter the overall amount of attention that might otherwise be devoted to the target, such diffusion could be expected to decrease target learning. On the contrary, the results showed that when choice was permitted, attention to both background and targets was enhanced relative to the force condition. In fact, taking this relationship one step further, it was found that the more contact with the background items, the better the target learning. This conclusion is based upon performance in the reject condition, in which subjects selected target items by explicitly rejecting the nondesired (background) items, presumably maximizing contact with the background items. Performance in the reject condition tended to be better than it was in the choice condition. Thus, these data, along with those of Schulman (1973), support the contention that the efficacy of target learning is a direct function of the amount of background or contextual learning. On the other hand, a motivational interpretation, such as that of Monty et al. (1979) and Perlmuter and Monty (1977), cannot be ruled out entirely. Support for this type of interpretation comes from the observation that the benefits of choice appear to be larger with the use of a between-subjects design than they are with a within-subjects design employed earlier (Perlmuter \& Monty, 1982). It is, of course, entirely possible that the opportunity to choose provides the motivational resources for increased attention. Thus both motivational and attentional factors may be responsible for the improvement in performance. This possibility warrants further research.

\section{REFERENCES}

LocAscio, D., \& LEY, R. Scale-rated meaningfulness of 319 cvcve word paralogs previously assessed for associative reaction time. Journal of Verbal Learning and Verbal Behavior, 1972, 11, 243-250.

Monty, R. A., Geller, E. S., Savage, R. E., \& Perlmuter, L. C. The freedom to choose is not always so choice. Journal of Experimental Psychology: Human Learning and Memory, 1979, 5, 170-178.

Perlmute r, L. C., \& Monty, R. A. The importance of perceived control: Fact or fantasy? American Scientist, 1977, 65, 759-765.

Perlmuter, L. C., \& Monty, R. A. Contextual effects on learning and memory. Bulletin of the Psychonomic Society, 1982, 20, 290-292.

Perlmuter, L. C., Monty, R. A., \& Kimble, G. A. Effect of choice on paired associate learning. Journal of Experimental Psychology, 1971, 91, 47-51.

Schulman, A. I. Recognition memory and the recall of spatial location. Memory \& Cognition, 1973, 1, 256-260.

(Received for publication October 29, 1982.) 